\title{
An interval type-2 FCM for color image segmentation
}

\author{
Abdullah Hamad*, Sadegh Aminifar and Muhammadamin Daneshwar \\ Department of Computer Science, Soran University, Erbil, Iraq
}

Received: 14-September-2019; Revised: 20-December-2019; Accepted: 23-December-2019

(C)2020 Abdullah Hamad et al. This is an open access article distributed under the Creative Commons Attribution (CC BY) License, which permits unrestricted use, distribution, and reproduction in any medium, provided the original work is properly cited.

\begin{abstract}
In today's digital life, the segmentation of images is a very important issue. Each image contains a big amount of data. The internal relation between data of one image is nonlinear and ambiguous. There is a big uncertainty to find all segments of an image. Therefore, there is a big necessity to find a segmentation method for handling high uncertainty. In this paper, some of the previous works that have done on image segmentation have been improved and extended. A novel fuzzy c-means (FCM) is applied for the segmentation of images that inherently have high uncertainty and vagueness. A new method is used based on interval type-2 fuzzy sets, and the idea of reducing higher-order sets to lower order to capture the uncertainty of the images based on the decisiveness method. The higher peak signal to noise ratio (PSNR) value and the Jaccard similarity value for colour images show better segmentation results for having better performance and better effects on segmenting real world images. The results show that the proposed algorithm handles the segmentation of colour images better than the previous type-1 and type-2 FCM.
\end{abstract}

\section{Keywords}

Fuzzy c-means algorithm, Image processing, Image segmentation, Uncertainty.

\section{Introduction}

In some image processing applications, segmenting the images is crucial and essential due to its usefulness for understanding images easily and extracting meaningful parts from them. The process is to divide images into different interesting sections. In this process numerous approaches have been introduced and studied in the literature, like histogram Thresholding [1, 2], Edge Detection [3, 4], Region Growing [5, 6], Watershed Transformation $[7,8]$. In the most recent decade fuzzy techniques are introduced in several fields of image processing to process image data with vagueness and ambiguity characteristics. The fuzzy c-means (FCM) algorithm is a popular technique that is widely used in the field of segmentation [9-11]. But it is founded that with type-1 fuzzy sets, ambiguity might not be taken well in the images because of the complexity of the image visual structures. Besides, with ordinary type-1 fuzzy sets, satisfactory results might not be obtained, so using, the type-2 fuzzy logic would be necessary to manage uncertainty exists in real-world problems.

*Author for correspondence

12
1.1Type- 1 and type-2 fuzzy sets

The fuzzy logic systems are based on the fuzzy sets. Fuzzy sets are sets with elements that can only participate with a certain degree of membership function. Therefore, a fuzzy set does not have a crisp boundary. When the universe, $D_{x}$, is continuous and infinite, a fuzzy set $X$ in $D_{x}$ can be defined type- 1 and typ-2 fuzzy logic as follows respectively:

$X=\left\{\left(x, \mu_{x}(x)\right) \mid x \in D_{x}\right\}$

$\tilde{X}=\left\{\left((x, u), \mu_{\tilde{A}}(x, u) \mid \forall x\right.\right.$

$\left.\in X \forall u \in J_{x} \subseteq[0,1]\right\}$

T2FSs are the continuation of type-1 [12, 13], they extended to provide additional information in the secondary membership function, which they are not only depend on a variable, it also depends on variable $u$.

\subsection{Type-1 FCM}

Fuzzy c-means [14] is one of the clustering algorithms that group data objects based on similarity, in image processing, FCM divides pixels in images to different segments based on their contribution degrees to different segments. One of the popular and powerful techniques used in cluster 
analysis and medical imaging is FCM. the algorithm works in step by step procedure it forms a fuzzy partition matrix during its implementation and also requires a cluster center along with objective function. The Equation 3 defines the objective function of the algorithm, its value should be decreased in any step of its execution.

$J_{m}=\sum_{i=1}^{N} \sum_{j=1}^{C} \mu_{i j}^{m}\left\|x_{i}-c_{j}\right\|^{2}$

FCM objective function decreases subject to constraints of membership grades, we can calculate membership values $\mu_{i j}$ and update cluster centres $c_{j}$ as follows:

$$
\begin{aligned}
\mu_{i j}= & \frac{1}{\sum_{k=1}^{C}\left(\frac{d_{j i}^{2}}{d_{k i}^{2}}\right)^{\frac{2}{m-1}}} \\
& \quad \text { for } i \in\{1, \ldots, N\}, j \in\{1, \ldots \ldots, C\} \\
C_{j}= & \frac{\sum_{i=1}^{N} \mu_{i j}^{m} x_{i}}{\sum_{i=1}^{N} \mu_{i j}^{m}}, \text { for } j=1 \ldots C
\end{aligned}
$$

The algorithm execution steps will stop when $\|U(k+1)-U(k)\|<\varepsilon$. when $\varepsilon$ is some criteria for the termination of the algorithm, its value is always between 0 and 1 , and $\mathrm{k}$ represents the repetition steps.

\subsection{Interval type-2 FCM}

Hwang and Rhee [15] introduced the interval type-2 fuzzy FCM method as enhanced method for capturing and managing high uncertainty, they have worked on managing uncertainty in the field of pattern recognition focusing on FCM algorithm. They extended a pattern set in to an interval type 2 fuzzy sets (IT2FS) by involving two different values of fuzzifier $\mathrm{m}\left(m_{1}\right.$ and $\left.m_{2}\right)$ which creates a foot of uncertainty (FOU) for the fuzzifier and modified the procedure of FCM. IT2 FCM has two membership functions, upper and lower memberships and it has two fuzzifier values $m_{1}$ and $m_{2}$, representing different fuzzy degrees and providing different objective functions as listed in the Equation 6 and Equation 7:

$J_{m 1}=\sum_{i=1}^{N} \sum_{j=1}^{C} \mu_{i j}^{m_{1}}\left\|x_{i}-c_{j}\right\|^{2}$

$J_{m 2}=\sum_{i=1}^{N} \sum_{j=1}^{C} \mu_{i j}^{m_{2}}\left\|x_{i}-c_{j}\right\|^{2}$

Both upper and lower interval type-2 fuzzy membership functions can be calculated using the Equation 8 and Equation 9.

Upper membership function:

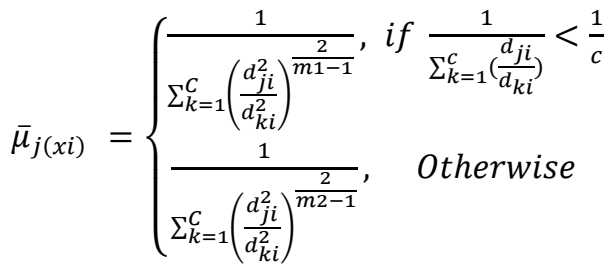

Lower membership function

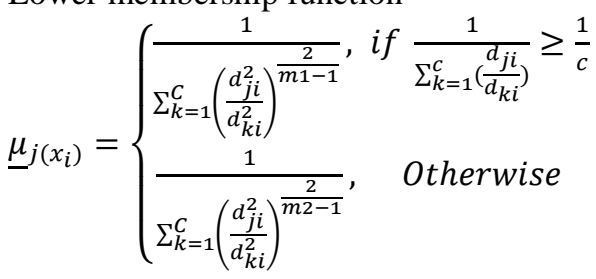

The foot of uncertainty of fuzzifier $m$ is obtained by the two fuzzifiers $\mathrm{m} 1$ and $\mathrm{m} 2$.

\section{Our proposed method}

In our proposed method for image segmentation, we used IT2FCM for colour image segmentation with some modification, we employed a filtering step into the process of segmentation because FCM and IT2FCM are both sensitive to noises, median filter [16] which is a none-linear type filter is used as a first step for eliminating noises from images especially impulse noises, none-linear filters are good due to their robustness for removing noise pixels without blurring edges. In this paper, the interval type-2 fuzzy sets are used as basic membership functions in fuzzy clustering. The type reduction which is applied to used interval type-2 fuzzy membership functions is decisiveness method which is proposed in [17] and [18]. In decisiveness type reduction method, the interval type- 2 is reduced to type one using the below equation for calculating $\mu_{d}$ as reduced type membership degree:

$\mu_{\mathrm{d}}=\mu_{\mathrm{p}}-\left(\frac{1}{2}\right){\underset{\sim}{\mathrm{u}}}^{2}$

In which: $\mu_{p}=\frac{\mu_{j}^{R}\left(x_{i}\right)+\mu_{j}^{L}\left(x_{i}\right)}{2}$ and $\underset{\sim}{\mathrm{u}}=\frac{\mu_{j}^{R}\left(x_{i}\right)-\mu_{j}^{L}\left(x_{i}\right)}{2}$

One of the advantages of this determiner is that the more the uncertainty bandwidth increases, the determined value of a point decreases in a linear manner. For instance, if $\underset{\sim}{\mathrm{u}}=1$, then $\mu_{\mathrm{dtr}}=0$ and if $\underset{\sim}{\mathrm{u}}=0$, then $\mu_{\mathrm{dtr}}=1$ and its fluctuations between these two points is in accordance with u. After choosing a decisiveness type reduction method which has a powerful effect on uncertainty handling, the applied algorithm for image segmentation is proposed as below: 
Steps of our IT2FCM method for colour image segmentation

Step 1) Apply median filtering image to input images.

Step 2) Set the number $c$ of the cluster prototypes $(2 \leq c \leq N)$ and fuzzifiers $\mathrm{m}, m_{1}$ and $m_{2}$. Set $\varepsilon>0$ to a very small value and initialize the cluster center $V$ randomly.

Step 3) Estimating FCM membership $\mu_{i j}$ using $m 1$ and $m 2$ in the following orders:

- Compute the distance between input patterns and cluster centers $d_{i k}^{2}=\left\|x_{k}-v_{i}\right\|$ (where $\left\|x_{k}-v_{i}\right\|$ is the euclidean norm).

$$
\begin{aligned}
& \text { - } \mu_{i j}=\frac{1}{\sum_{k=1}^{C}\left(\frac{x_{i j}^{2}}{x_{i k}^{2}}\right)^{\frac{2}{m-1}}} ; \quad i \in\{1, \ldots . ., N\}, j \in \\
& \{1, \ldots \ldots, C\}
\end{aligned}
$$

Step 4) Calculating The upper and lower membership functions $\bar{\mu}_{i j}^{(1)}, \underline{\mu}_{i j}^{(1)}$ using Equations 8 and 9.

Step 5) Updating cluster centers $V^{l+1}$ using Equation 10.

$v_{j}=\frac{v_{L}+v_{R}}{2}$

Step 6) Applying decisiveness type-reduction as follows in Equation 12:

$\mu_{j}\left(x_{i}\right)=\frac{\mu_{j}^{R}\left(x_{i}\right)+\mu_{j}^{L}\left(x_{i}\right)}{2}-\left(\frac{\mu_{j}^{R}\left(x_{i}\right)-\mu_{j}^{L}\left(x_{i}\right)}{2}\right)^{2}, j=$

$1, \ldots, C$

\section{Experimental results}

I have tested FCM and our proposed algorithm on randomly chosen images from the publicly-available ground-truth segmentation databases, like BSDS500 from the Berkeley Segmentation Dataset and Benchmark as showed some image examples from the dataset in Figure 1. Both algorithms are simulated using MATLAB 2016b. A $3 \times 3$ kernel window is used for median filtering. And the fuzzifier parameters are set like: $\mathrm{m}=3, \mathrm{~m} 1=2, \mathrm{~m} 2=$ 7.

In our experiments, we have used two clustering validation methods partition coefficient (PC) and partition entropy (PE) as formulated in Equations 13 and 14 respectively [19]. We also compared the quality of segmentation results of both FCM and IT2FCM by using Jaccard similarity coefficient and peak signal to noise ratio (PSNR) methods [20]. The Jaccard similarity coefficient that is also called

Table 1 Numerical comparisons using validation methods 14 intersection over union is defined in Equation 15, and PSNR is defined in Equation 16.

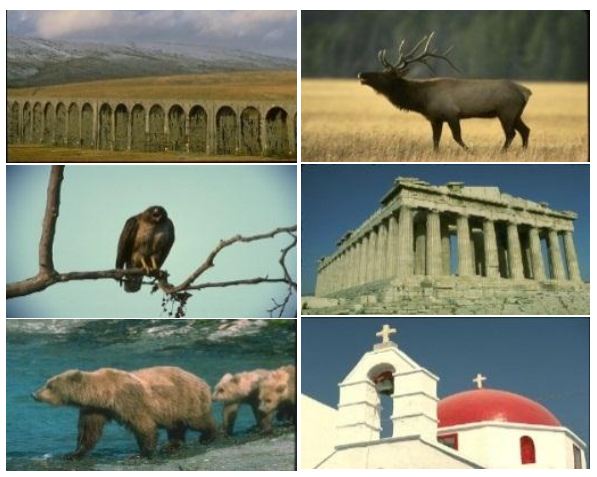

Figure 1 Example of images from the BSDS500 dataset

$P C=\frac{1}{N} \sum_{i=1}^{c} \sum_{j=1}^{N} \mu_{i j}^{2}$

$P E=-\frac{1}{N} \sum_{i=1}^{c} \sum_{j=1}^{N} \mu_{i j} \log _{a} \mu_{i j}$

$I o U=\frac{\text { GroundTruth }(I) \cap \text { segmented }(I)}{\text { GroundTruth }(I) \cup \text { segmented }(I)}$

$P S N R=10 \log _{10}\left(\frac{255^{2}}{M A E}\right)$

Where MAE is the mean absolute error of the segmented image computed as in Equation 17.

$M A E=\frac{\sum_{M, N}\left[I_{1}(m, n)-I_{2}(m, n)\right]^{2}}{M * N}$

Where $I_{1}(m, n)$ is the segmented image and $I_{2}(m, n)$ is the original image.

\section{Discussion}

For the validation clustering methods, the clustering algorithm gets the best result when the Partition coefficient is maximum and the partition entropy is the minimum. As we compared both methods in Table 1, it indicates that IT2FCM has a better performance in dealing with the uncertainties and obtained better cluster results.

For measuring quality results, the higher the PSNR value means better segmentation results and the Jaccard similarity value should be higher and close to 1 as possible. The Jaccard index, also known as the intersection over union and the Jaccard similarity coefficient, is a statistic used for gauging the similarity and diversity of images. Table 2 shows the segmentation performance between the two algorithms. It indicates that our proposed method has a better performance than the conventional type- 1 FCM. 


\begin{tabular}{lllll}
\hline Images & FCM & PE & IT2FCM & PC \\
\hline 1 & PC & 0.4745 & 0.8562 & 0.2516 \\
2 & 0.7370 & 0.4546 & 0.8125 & 0.3327 \\
3 & 0.7466 & 0.4572 & 0.7804 & 0.3999 \\
4 & 0.7429 & 0.4769 & 0.7934 & 0.3730 \\
5 & 0.7373 & 0.3324 & 0.8456 & 0.2948 \\
6 & 0.8124 & 0.5633 & 0.6956 & 0.5471 \\
7 & 0.6848 & 0.3366 & 0.8651 & 0.2789 \\
8 & 0.8294 & 0.3349 & 0.8282 & 0.3101 \\
9 & 0.8254 & 0.4844 & 0.9284 & 0.1395 \\
10 & 0.7382 & 0.1579 & 0.9455 & 0.1007 \\
\hline
\end{tabular}

Table 2 Performance measures of FCM and IT2FCM

\begin{tabular}{|c|c|c|c|c|}
\hline \multirow[b]{2}{*}{ Images } & \multicolumn{2}{|l|}{ FCM } & \multicolumn{2}{|c|}{ IT2FCM } \\
\hline & IoU & PSNR & IoU & PSNR \\
\hline 1 & 0.7599 & 20.3652 & 0.8378 & 22.7495 \\
\hline 2 & 0.6502 & 7.2874 & 0.7048 & 26.0539 \\
\hline 3 & 0.0607 & 16.8082 & 0.1229 & 26.5431 \\
\hline 4 & 0.3449 & 11.8847 & 0.6551 & 24.3710 \\
\hline 5 & 0.5248 & 10.3524 & 0.7371 & 24.5885 \\
\hline 6 & 0.5062 & 4.6628 & 0.5473 & 25.5365 \\
\hline 7 & 0.7207 & 6.7526 & 0.8770 & 21.9642 \\
\hline 8 & 0.5576 & 5.2015 & 0.8019 & 21.4530 \\
\hline 9 & 0.6326 & 17.5722 & 0.9236 & 25.6367 \\
\hline 10 & 0.6246 & 6.2465 & 0.8467 & 25.2261 \\
\hline
\end{tabular}

We also provide some examples of the segmentation results using IT2FCM in Figure 2.

The approach of measuring the similarity between two images is using PSNR and Jaccard parameters: An image is selected, to be more correct, a region-ofinterest in each image then the pixel features are identified for comparing (e.g., red, blue, green and gradient orientation). Finally, similarity formula is set up. The results which are shown in Table 2 shows higher PSNR and Jaccard compare to their type-1 and traditional type- 2 methods. Figure 2 confirms this optimization.

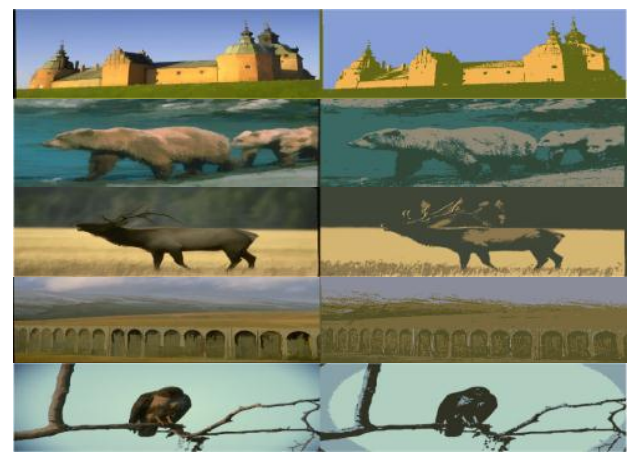

Figure 2 Segmentation results using IT2FCM, left original images, and right are the segmentation results

\section{Conclusion}

In this paper, an image segmentation technique based on FCM is enhanced and improved in term of handling uncertainty. Interval type-2 fuzzy sets and decisiveness type reduction methods are used in the proposed technique. Type-1 FCM algorithm and proposed algorithm are tested on different kinds of color images. The performance of the proposed algorithm for segmentation has been compared with previous type-1 FCM algorithm. The objective evaluation metrics like partition coefficient (PC) and partition entropy (PE) are used in their comparisons. Other performance measures (Jaccard similarity coefficient, and Peak signal to noise ratio) are also used to test the algorithms. Results show that our interval type-2 algorithm are better than the type-1 FCM algorithm and applying decisiveness method for type reduction of interval type-2 provides an active method for handling uncertainty in image segmentation.

\section{Acknowledgment}

None.

\section{Conflicts of interest}

The authors have no conflicts of interest to declare. 


\section{References}

[1] Mirkazemi A, Alavi SE, Akbarizadeh G. Fast image segmentation based on adaptive histogram thresholding. In the international symposium on artificial intelligence and signal processing 2015 (pp. 165-9). IEEE.

[2] Bhakat S, Periannan S. Brain tumor detection using cuckoo search algorithm and histogram thresholding for MR images. In smart innovations in communication and computational sciences 2019 (pp. 85-95). Springer, Singapore.

[3] Salman NH, Ghafour BM, Hadi GM. Medical image segmentation based on edge detection techniques. Advances in Image and Video Processing. 2015; 3(2):1-9.

[4] Senthilkumar R, Bharathi A, Sowmya B, Sugunamuki KR. Image segmentation edge detection techniques using-soft computing approaches. In international conference on soft-computing and network security 2018 (pp. 1-6). IEEE.

[5] Kamdi S, Krishna RK. Image segmentation and region growing algorithm. International Journal of Computer Technology and Electronics Engineering. 2012; 2(1):103-7.

[6] Borges VR, De Oliveira MC, Silva TG, Vieira AA, Hamann B. Region growing for segmenting green microalgae images. IEEE/ACM Transactions on Computational Biology and Bioinformatics. 2016; 15(1):257-70.

[7] Kornilov A, Safonov I. An overview of watershed algorithm implementations in open source libraries. Journal of Imaging. 2018; 4(10):1-15.

[8] Shabbir H, Bibi H, Aamir S, Saqib MS, Afshan MN. Image segmentation using marker-controlled watershed transformation and morphology. Research Journal of Computer Science and Information Technology. 2018; 2(1):55-60.

[9] Naz S, Majeed H, Irshad H. Image segmentation using fuzzy clustering: a survey. In international conference on emerging technologies 2010 (pp. 181-6). IEEE.

[10] Aljebory KM, Mohammed TS. Developing modified fuzzy c-means clustering algorithm for image segmentation. In international multi-conference on systems, signals \& devices 2018 (pp. 1221-7). IEEE.

[11] Adhikari SK, Sing JK, Basu DK, Nasipuri M. Conditional spatial fuzzy c-means clustering algorithm for segmentation of MRI images. Applied Soft Computing. 2015; 34:758-69.

[12] Mendel JM. Uncertain rule-based fuzzy systems. In introduction and new directions 2017 (p. 684). Springer International Publishing.

[13] Ruiz-Garcia G, Hagras H, Pomares H, Rojas I. Towards a fuzzy logic system based on general forms of interval type-2 fuzzy sets. IEEE Transactions on Fuzzy Systems. 2019:27(12):2381-95.
[14] Bezdek JC, Ehrlich R, Full W. FCM: the fuzzy cmeans clustering algorithm. Computers \& Geosciences. 1984; 10(2-3):191-203.

[15] Hwang C, Rhee FC. Uncertain fuzzy clustering: interval type-2 fuzzy approach to \$ c \$-means. IEEE Transactions on Fuzzy Systems. 2007; 15(1):107-20.

[16] George G, Oommen RM, Shelly S, Philipose SS, Varghese AM. A survey on various median filtering techniques for removal of impulse noise from digital image. In conference on emerging devices and smart systems 2018 (pp. 235-8). IEEE.

[17] Aminifar S, Marzuki A. Uncertainty in interval type-2 fuzzy systems. Mathematical Problems in Engineering. 2013.

[18] Aminifar S, Bin Marzuki A. Horizontal and vertical rule bases method in fuzzy controllers. Mathematical Problems in Engineering. 2013.

[19] Halkidi M, Batistakis Y, Vazirgiannis M. On clustering validation techniques. Journal of Intelligent Information Systems. 2001; 17(2-3):107-45.

[20] Sumithra MG, Deepa B. Performance analysis of various segmentation techniques for detection of brain abnormality. In region 10 conference 2016 (pp. 205661). IEEE.

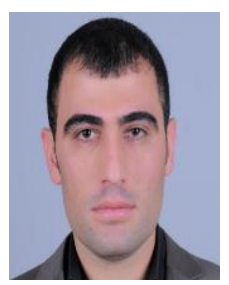

Abdullah Shekha Hamad received a Bachelor's degree in Computer Science at the University of Sulaimani, Sulaimania, Iraq (2010). He also got his MSc in Artificial Intelligence at Soran University, Soran, Erbil, Iraq (2019). His research interests are Image Processing, Computer Vision, and Recommender Systems.

Email: abdullah.shekha86@gmail.com

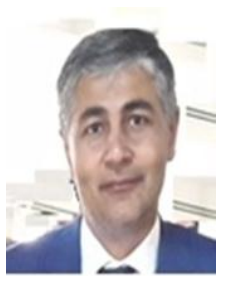

Sadegh Abdollah Aminifar received $\mathrm{BSc}$ in Electronics from Shahid Beheshti University, Tehran, Iran, 1999. He received MSc in Digital Fuzzy System Chip Design and Implementation from Urmia University, Urmia Iran, 2002. He also got his Ph.D. from University Science Malaysia in designing and implementation of fuzzy systems (Soft Computing) for handling uncertainty in industrial applications, Penang, Malaysia (2014). His interested fields are Digital Systems, Fuzzy Systems, and Image Processing. He has recently concentrated on research on Image Processing as an Assistant Professor at Soran University, Soran, Erbil, Iraq Email: sadiq.aminifar@soran.edu.iq 


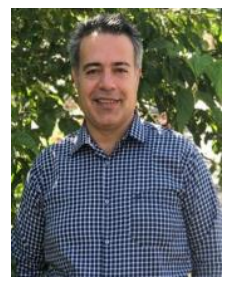

Muhammadamin Daneshwar is currently an Assistant Professor at Soran University, Soran, Kurdistan, Iraq. He received his BSc in Electrical and Electronics Engineering from Urmia University, Urmia, Iran (2001), and also received MSc in Electronics in 2007. He got his Ph.D. degree from the school of Electrical and Electronics Engineering at University Sains Malaysia (USM) in 2016. His field is Computational Intelligence. He is also interested in Neural and Fuzzy System, System Identification, and Fault Detection and Diagnosis.

Email: muhamad.muhamad@soran.edu.iq 